\title{
RENAL DYSFUNCTION IN MALARIA INFECTION AROUND NAVI MUMBAI
}

\section{YADAV K.S. ${ }^{*}$, SMITA PATIL ${ }^{2}$, REKHA BHAGWAT ${ }^{1}$, RAVISEKHAR K. ${ }^{3}$, JOY GHOSHAL ${ }^{4}$, SHIRISH PATIL ${ }^{5}$ AND MILIND HANCHATE 6}

\author{
1Department of Biochemistry, Padmashree Dr. D.Y. Patil Medical College, , Navi Mumbai-706 \\ 2,Department of Medicine, Padmashree Dr. D.Y. Patil Medical College, , Navi Mumbai-706 \\ 3Department of Microbiology, Padmashree Dr. D.Y. Patil Medical College, Navi Mumbai-706 \\ ${ }^{4}$ Department of Anatomy, Padmashree Dr. D.Y. Patil Medical College, Navi Mumbai-706 \\ 5Department of PSM, Padmashree Dr. D.Y. Patil Medical College, Navi Mumbai-706 \\ 6Department of PSM, Shantiram Medical College, Nandyal- 518501 \\ ${ }^{*}$ Corresponding Author: Email- ksy_rahul@rediffmail.com
}

Received: December 12, 2011; Accepted: January 15, 2012

\begin{abstract}
Introduction: Acute renal failure (ARF) is seen mostly in Plasmodium falciparum infection, but $P$. vivax and P. malariae can cause renal impairment rarely. Malarial ARF is commonly found in adults and older children with falciparum malaria.

Material \& Methods: Ninety five patients from Pad. Dr. D Y Patil Hospital and Research Centre, Navi Mumbai, were included in this study during July-Aug 2010. Out of 95 patients 38 patients are control, 10 patients infected by $P$. falciparum, 36 patients infected by $P$. vivax and 11 are mix infection of $P$. falciparum \& P. vivax. All patients' diagnosis is confirmed by clinical examination as well as peripheral blood smear.

Results: Renal involvement present as electrolyte abnormality as Hyponatraemia, Oliguria, Uraecemia and jaundice. Acute Renal Failure (ARF) occurs as a complication of P. falciparum malarial infection in less than $1 \%$ of cases, but the mortality reported up to $40 \%$. Malarial ARF is diagnosed when serum creatinine level greater than $3 \mathrm{mg} / \mathrm{dL}$ and/or urinary output is less than $400 \mathrm{ml}$ in 24 hours. The serum concentrations of Creatinine, urea, proteins ( Total proteins \& albumin), Bilirubin (conjugated and total bilirubin) in malaria patients were significantly higher $(p<0.05)$ than those of malaria free individuals. We conclude that renal dysfunction, acute renal failure and liver dysfunction are clinical features of malaria

Conclusion: In spite of several researches and ultramodern techniques, mechanism of malarial ARF and its effective management has remained unclear. In many cases reversal of renal dysfunction takes place may be due to biotransformation of antigen and response of immune system. In addition, the literature is almost silent on the mechanism of recent increase in incidence of ARF and multiple complications specifically around coastal regions in India.
\end{abstract}

Key words- renal dysfunction, biochemical parameters, plasmodium falciparum, plasmodium vivax

Citation: Yadav KS, et al. (2012) Renal Dysfunction in Malaria Infection around Navi Mumbai. International Journal of Medical and Clinical Research, ISSN: 0976-5530 \& E-ISSN:0976-5549, Volume 3, Issue 1, 2012, pp-110-114.

Copyright: Copyright@2012 Yadav KS, et al. This is an open-access article distributed under the terms of the Creative Commons Attribution License, which permits unrestricted use, distribution, and reproduction in any medium, provided the original author and source are credited.

\section{Introduction}

Malaria has emerged as one of the top 10 killer diseases around the globe. It is the major cause of mortality in various tropical and subtropical regions. Approximately half of the world population is vulnerable to malaria, may be more than that. More than 500 million people reported positive cases of malaria and leading to death in 2 to 3.0 million cases. Majority of the malarial infected cases as well as deaths occur in sub-Saharan Africa. Outside Africa, the disease is seen in about 100 countries, Indian subcontinent and Brazil contributing nearly two thirds of these cases. Acute renal failure (ARF) occurs as a complication of plasmodium falciparum malaria in less than $1 \%$ of cases, but the mortality in these cases may be up to $40 \%$. Malaria is a highly destructive disease in humans caused by a protozoan, plasmodium species. It accounts for an estimated 2-3 million deaths annually across over 100 countries. [1] Malarial infection is caused by four species of the genus Plasmodium namely, Plasmodium vivax, P. falciparum, P. malariae and $P$. ovale. Out of the species of plasmodium parasite, plasmodium falciparum, plasmodium vivax, plasmodium malariae and plasmodium ovale that causes malaria in humans. Plasmodium falci- 
parum is responsible for most deaths and most of the severe complications although renal involvement is also known to be caused by plasmodium malariae.[2-5] Cases of malarial infection associated renal and hepatic impairment have been reported from different parts of malaria endemic countries.[6-11] The severity of malaria infection associated renal impairment in a particular region is largely a function of the disease spread in the region and other a etiological factors prevailing in the area.[12] It is more common in adults than children. Malarial acute renal failure is diagnosed when serum creatinine level elevated above $3 \mathrm{mg} / \mathrm{dL}$ and/or when urinary output is less than $400 \mathrm{ml}$ in a day.

Common clinical presentations of infection with all four Plasmodium species are chills, rigors, sweating, body aches, headache, nausea and general weakness Severe life-threatening complications such as cerebral malaria (CM), severe anemia, acidosis, respiratory distress syndromes (RDS), jaundice, acute renal failure (ARF),acute respiratory distress syndrome (ARDS), etc. occur mostly with plasmodium falciparum infection. A few studies have appeared indicating association of severe complications of malaria with plasmodium vivax infection [13-15]. Renal involvement has been reported in plasmodium falciparum plasmodium malariae, and recently in plasmodium vivax infections plasmodium malariae associated nephropathy was reported mainly from Africa, that too before 1980. Earlier literature on P. vivax renal failure are too less and inconclusive to merit a detailed review.

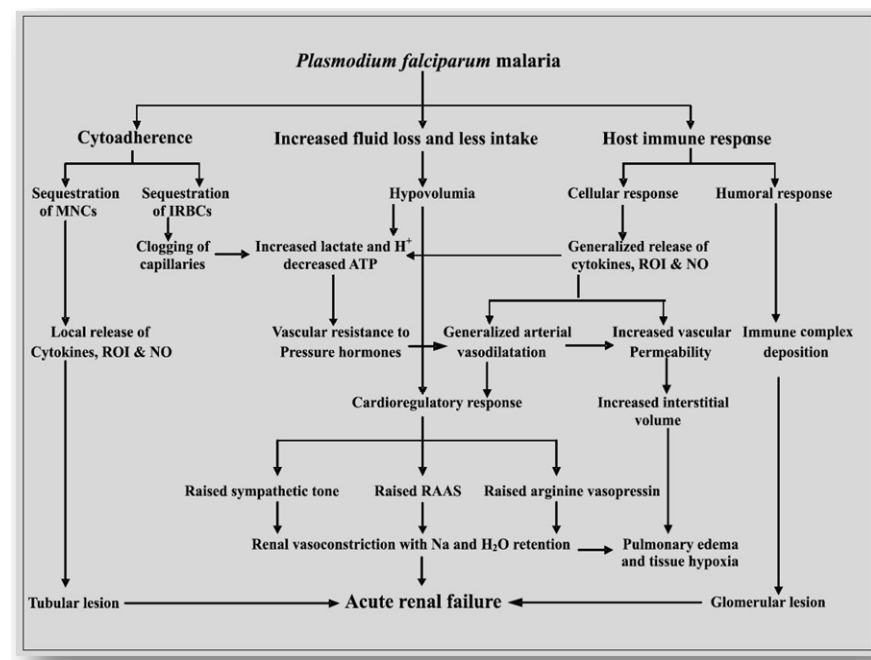

Fig.1- Etiology of Falciparum Malaria infection causes damage to kidney. (Adapted from J Vector Borne Dis 45,2008, pp. 83-97)

\section{Abbreviations:}

MNCs-Mononuclear cells; RBCs-Infected red blood cells;

RAAS- Renin-angiotensin-aldosterone system; ROI-Reactive oxygen intermediates; NO- Nitric oxide; H+- Hydrogen ion

Acute renal failure (ARF) occurs as a common complication of plasmodium falciparum malaria in less than $1 \%$ of cases, but the mortality in these cases may be up to $40 \%$. As per the WHO criteria malarial acute renal failure is diagnosed when serum creatinine level rises above $3 \mathrm{mg} / \mathrm{dL}$ and/or when urinary output in 24 hours is less than $400 \mathrm{ml}$ [16]. Renal involvement varies from mild proteinuria to severe azotemia associated with metabolic acidosis. [17] Renal involvement in plasmodium malariae infection: Incidence of progressive glomerulonephritis was significantly higher in malariaendemic areas of Africa. Plasmodium malariae was considered an important cause of chronic renal dysfunction in malarial infection. [18]

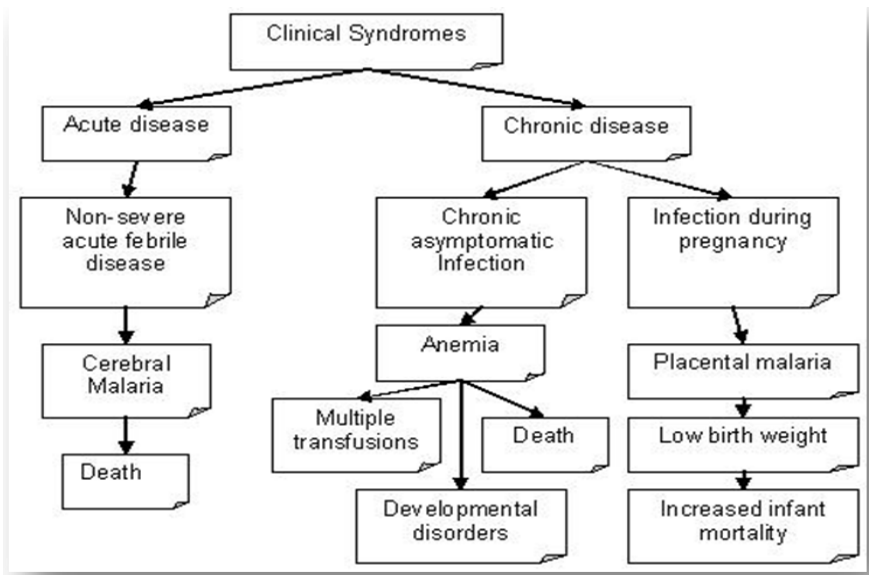

Fig. 2- Clinical syndromes of malaria (adopted from J. MacArthur, $\mathrm{CDC})$

Exact mechanism of renal failure in falciparum malaria is not clearly known. Several researchers hypotheses including mechanical obstruction by infected erythrocytes by the parasites, immune mediated glomerular pathology, fluid loss due to multiple other mechanisms and alterations in the renal microcirculation, etc.[3,19 -20] The incidence of glomerulonephritis gradually declined along with eradication of malaria in many parts of Africa.[21,22] The pathogenesis of renal involvement is possibly mediated through immune complex deposition. [23] The disease progresses to renal failure even after successful eradication of the infection may be due to their toxicity, exact mechanism is unclear. [21, 24] In plasmodium falciparum infection malarial acute renal failure (MARF) often occurs in association with signs and symptoms of multi-organ involvement, jaundice and thrombocytopenia are present in more than $70 \%$ of cases. $[3,25]$

\section{Material and Methods}

Ninety five patients attended IPD and OPD at Pad. Dr. D Y Patil Hospital and Research Centre, Navi Mumbai, were included in this study during peak season in July-Aug 2010. Out of 95 patients 38 patients are non-malarial, were taken as controls, 10 patients suffering with plasmodium falciparum, 36 patients suffering with plasmodium vivax and 11 are mix infection of plasmodium falciparum and plasmodium vivax. No other species of malaria included in this study due to very low incidence. All patients' diagnosis is confirmed by clinical examination as well as peripheral blood smear.

\section{Inclusion Criteria}

The following patients were included in present study

- Patients with ARF and peripheral smear positive for malaria parasite

- Absence of any other disease which leads to ARF.

\section{Exclusion Criteria}

The following patients were excluded from present study:

- patients who had hemolysis along with glucose-6-phosphate dehydrogenase deficiency 
- pregnant women,

- with high parasitaemia,

- with deep jaundice,

- with prolonged dehydration, and/or,

- patients receiving Nonsteroidal Antiinflammatory Drugs.

Lab. investigations

In addition to the Complete Blood Count (CBC) with peripheral smear study for malaria parasite, the following tests were performed for assessment of Kidney and Liver function.

Renal Function Test (RFT)

- Blood Urea (BU),

- Creatinine,

- Uric Acid( UA),

- Electrolytes $\left(\mathrm{Na}^{+} \& \mathrm{~K}^{+}\right)$

- Total Proteins and Albumin

Liver Function Test (LFT)

- Total ,Direct and Indirect Bilirubin,

- $\quad$ SGPT(ALT),

- $\operatorname{SGOT}(\mathrm{AST})$,

- Total Protein and Albumin.

\section{Results\& Statistical Analysis} Summary of results:

Table 1-Result of all renal parameters \& platelet count in all four study groups

\begin{tabular}{|c|c|c|c|c|c|c|c|c|c|}
\hline Group & DATA & BU & CREAT & UA & TP & ALB & $\mathrm{Na}^{+}$ & $\mathrm{K}^{+}$ & PLT \\
\hline \multirow{4}{*}{$\begin{array}{l}P V \\
n=36\end{array}$} & Count & 36 & 36 & 36 & 36 & 36 & 36 & 36 & 36 \\
\hline & Sum & 615 & 42.4 & 210.3 & 253.6 & 115.7 & 4892 & 132.31 & 3264 \\
\hline & Average & 17.08 & 1.17 & 5.847 & 7.04 & 3.29 & 135.88 & 3.67 & 93.27 \\
\hline & Variance & 193.3 & 0.362 & 5.7191 & 0.940 & 0.646 & 12.158 & 0.281 & 4813.25 \\
\hline \multirow{5}{*}{$\begin{array}{l}P F \\
n=10\end{array}$} & Count & 10 & 10 & 10 & 10 & 10 & & 10 & 10 \\
\hline & Sum & 143 & 10.3 & 46 & 60.7 & 24.8 & 1350 & 34.48 & 453 \\
\hline & Average & 14.3 & 1.03 & 4.6 & 6.07 & 2.48 & 135 & 3.44 & 50,33 \\
\hline & Variance & 35.56 & 0.020 & 0.98889 & 1.080 & 0.208 & 31.555 & 0.118 & 511.5 \\
\hline & Count & 11 & 11 & 11 & 11 & 11 & 11 & 11 & 11 \\
\hline \multirow{3}{*}{$\begin{array}{l}\text { PVPF } \\
\mathrm{n}=11\end{array}$} & Sum & 168 & 124 & 56.7 & 776 & 32 & 1493 & 36.8 & 559 \\
\hline & Average & 15.27 & 1.12 & 5.155 & 7.05 & 2.90 & 135.72 & 3.34 & 55.9 \\
\hline & Variance & 28.81 & 0.228 & 3.7467 & 1.468 & 0.199 & 9.818 & 0.078 & 894.544 \\
\hline \multirow{4}{*}{$\begin{array}{l}\text { Control } \\
n=38\end{array}$} & Count & 38 & 38 & 38 & 38 & 38 & 38 & 38 & 38 \\
\hline & Sum & 420 & 32.2 & 188.6 & 273.6 & 118.6 & 5174 & 146.6 & 7022 \\
\hline & Average & 11.05 & 0.84 & 4.96 & 7.2 & 3.12 & 136.11 & 3.85 & 189.78 \\
\hline & Variance & 44.10 & 0.101 & 4.3083 & 0.7276 & 0.3762 & 18.460 & 0.294 & $\begin{array}{l}16804.0 \\
9\end{array}$ \\
\hline
\end{tabular}

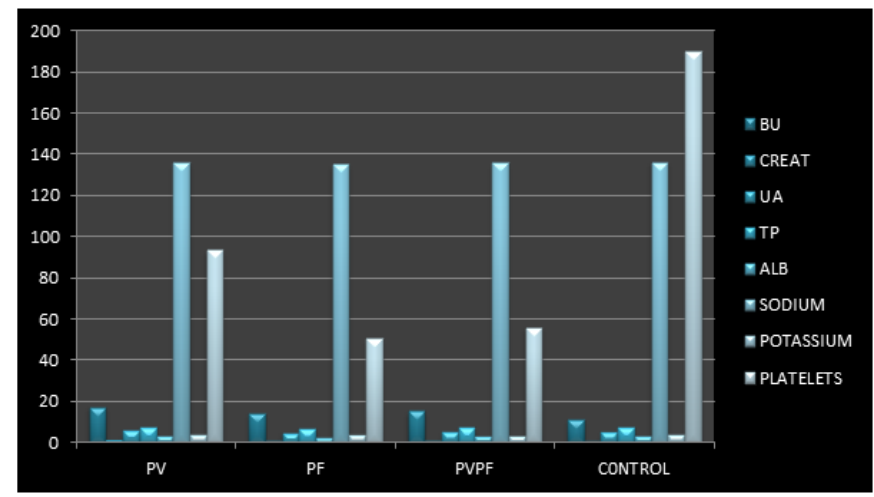

Fig. 3- Bar diagram of average value of all parameters in all study groups.
Table 2- Gender wise characteristic of the 57 study patients

\begin{tabular}{|c|c|c|c|c|c|}
\hline \multirow{2}{*}{$\begin{array}{l}\text { Sr } \\
\text { No }\end{array}$} & \multirow[t]{2}{*}{ Variable } & \multicolumn{2}{|c|}{ Male, $n=35$} & \multicolumn{2}{|c|}{ Female, $n=22$} \\
\hline & & $\begin{array}{l}\text { Total } \\
\text { cases }\end{array}$ & Percentage & $\begin{array}{l}\text { Total } \\
\text { cases }\end{array}$ & Percentage \\
\hline 1 & Fever & 32 & 91.42 & 21 & 95.45 \\
\hline 2 & Headache & 33 & 94.28 & 20 & 90.90 \\
\hline 3 & Thrombocytopenia & 34 & 97.14 & 22 & 100 \\
\hline 4 & Hyponatraemia & 11 & 31.42 & 8 & 36.36 \\
\hline 5 & Hyperbilirubinemia & 23 & 65.71 & 6 & 27.27 \\
\hline 6 & Oliguria & 19 & 54.28 & 5 & 22.72 \\
\hline 7 & Uraecemia & 16 & 45.71 & 5 & 22.72 \\
\hline
\end{tabular}

Fig. 4- Bar diagram of gender wise characteristics' of material patients.

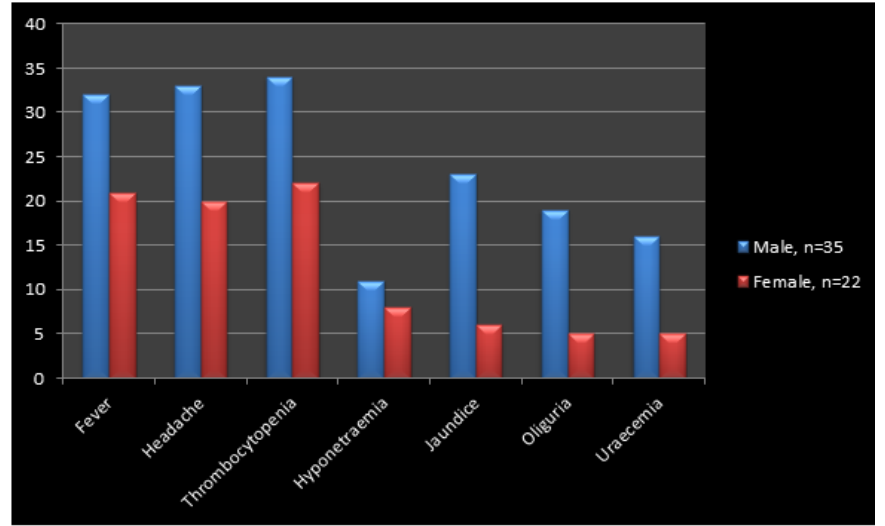

Table 3- Statistical analysis of renal parameters \& platelet count by ANOVA: Single Factor within group

\begin{tabular}{|lcccll|} 
Parameters & F Value & P value & F crit & Significance & $\begin{array}{l}\text { Null hypothe- } \\
\text { sis }\end{array}$ \\
Blood Urea & 2.318307 & 0.080721 & 2.704703 & No significance & Do not reject \\
Creatinine & 3.454287 & 0.019729 & 2.704703 & Significance & Reject \\
Uric Acid & 1.492131 & 0.221981 & 2.704703 & No significance & Do not reject \\
Total Protein & 3.698244 & 0.014589 & 2.704703 & Significance & Do not reject \\
Albumin & 3.458092 & 0.019636 & 2.704703 & Significance & Reject \\
Sodium & 0.222523 & 0.080524 & 2.704703 & Significance & Do not reject \\
Potassium & 3.987165 & 0.010215 & 2.704703 & Significance & Reject \\
Platelets & 10.71244 & 0.000004 & 2.709402 & Significance & Reject \\
\hline
\end{tabular}

In this study one-way ANOVA statistical package is used to compare means of study groups for numerical data [26].The ANOVA tests the null hypothesis that samples in two or more groups are drawn from the same population. The group means calculated from the same population, the variance between the group means should be lower than the variance of the samples. A higher ratio therefore implies that the samples were drawn from different populations [27].

An F-test is test in which an F-distribution under the null hypothesis the name was coined by George W. Snedecor, in honour of Sir Ronald A. Fisher. [28] F is the value of the test statistic and Fcrit is the critical value. The test statistic in ANOVA is the ratio of two scaled sums of squares reflecting different sources of variability. That is, $\mathrm{F}=$ Explained variance / Unexplained variance. The critical value is the number that the test statistic must exceed to reject the test. If $F>F$ crit, we reject the null hypothesis and if $F<F$ crit, we do not reject the null hypothesis. For example, the null hypothesis implies there is no relationship between two measured phenomena. [29] 


\section{Discussion}

Renal involvement in plasmodium vivax malaria: Renal involvement in plasmodium vivax malaria has been reported from Indian subcontinent. In one of the earlier studies, Mehta et al15 observed that out of 24 patients of malarial ARF, 16 were infected with plasmodium falciparum, 3 with plasmodium vivax, and 5 with mixed infection of plasmodium falciparum and plasmodium vivax. In a retrospective analysis, 13 of the 93 patients of malarial ARF had plasmodium vivax infection, while another 6 had mixed infection with plasmodium falciparum and plasmodium vivax. In present study we studied 57 patients infected by different species of malarial parasites. Out of 57,36 had plasmodium vivax, 10 had plasmodium falciparum and 11 had mixed infection of plasmodium falciparum \& plasmodium vivax. Evidence of renal involvement was noticed in 5 cases of plasmodium vivax malaria from a total of 57 cases.

Renal involvement in plasmodium falciparum malaria can present as electrolyte abnormality, abnormal urinary sediments and increased urinary protein excretion, high plasma Creatinine concentration, duration of ARF, etc. Mortality is higher when plasma Creatinine is high and urine output is low, delayed referral to the hospital, and when associated with other complications. Hyperbilirubinemia in falciparum malaria possibly predisposes for ARF, which may remain unnoticed sometimes. In patients of acute falciparum malaria with severe Hyperbilirubinemia may be the cause of ARF which was significantly associated with liver dysfunction as well. Thus the malarial infection specifically in case of plasmodium falciparum parameters are characterized by renal failure with oliguria and uraecemia associated with thrombocytopenia as well as $\mathrm{Hy}-$ ponatraemia.

Malarial ARF is suspected when urinary output falls to less than $400 \mathrm{ml}$ in 24 hours, which fails to improve after adequate rehydration. Occasionally malarial ARF may be non-oliguria in which case diagnosis can only be made from biochemical investigations that is $\mathrm{BU}$ and serum creatinine or more extensively 24 hours urine creatinine clearance test. The diagnosis is confirmed when the serum creatinine exceeds $3 \mathrm{mg} / \mathrm{dL}$ in adults and $1.5 \mathrm{mg} / \mathrm{dL}$ in children. Hyperbilirubinemia, Hyponatraemia and Thrombocytopenia were noted in majority of cases in both genders.

In acute renal failure, the glomerular filtration rate decreases over days to weeks. As a result, excretion of nitrogenous waste is reduced, and fluid and electrolyte balances cannot be maintained. Patients with acute renal failure are often asymptomatic, and the condition is diagnosed by observed elevations of blood urea nitrogen (BUN) and serum creatinine levels. Most authorities define the condition as an acute increase of the serum creatinine level from baseline i.e., an increase of at least $0.5 \mathrm{mg}$ per $\mathrm{dL}$. [30] Complete renal shutdown is present when the serum creatinine level rises by at least $0.5 \mathrm{mg}$ per $\mathrm{dL}$ per day and the urine output is less than $400 \mathrm{~mL}$ per day (oliguria). Blood Urea Nitrogen (BUN) is nonspecific indicator of renal function. [31] In critical patients BUN can elevate independently for various causes other than abnormal renal function. Thus changes in BUN do not convey degree of Uremia. Serum creatinine value is much more specific for assessing renal function than BUN. Several researchers earlier concluded that an inverse relationship between creatinine and ARF. $[30,32-34]$ The reason for this finding may be several extra-renal factors such as BMl, volume distribution of creatinine, creatinine production and liver abnormalities can alter creatinine level in critical ill patients. The clinician must cautious while interpreting serum creatinine value as single parameter in ARF associated with malaria.

Diagnosis of ARF based upon changes in serum creatinine may be delayed due to the fact that, in the non-steady-state conditions of ARF, as GFR falls creatinine secretion is increased. Large changes in GFR are initially manifested as small quantitative changes in serum creatinine in the first 24-48 hours after renal injury. After these one or two days, the degree of serum creatinine changes will reflect the change in GFR. Finally, the serum creatinine is stabilized, and that takes about 7 days. Therefore, it is almost impossible to exactly determine the onset of the ARF; and also calculating the exact time lapsed until the nephrology consultation. However, measuring serum creatinine level is a practical approach for discovering short-term alteration in renal function, despite its limitations, because it is readily used in clinical practice and it is specific for renal function.

Not all BUN and serum creatinine results values found rose in acute renal failure.

Around the globe where transmission of malaria is unstable and adults develop severe disease, acute renal failure with oliguria is relatively common and is a major contributor to mortality (Day et al., 1997; Stone et al., 1972). In another study renal failure is only rarely encountered as a major manifestation of severe malaria in semi-immune population of malaria-endemic African countries (Brewster and Greenwood, 1993; Weber et al., 1999). Polyuria with acute severe plasmodium falciparum malaria has not previously been described in adults and is not a feature of classic malaria. No significant associations were found with age and sex.

\section{Conclusion}

It is concluded that falciparum malaria in adults is one of the causes of acute renal failure in this population. In the patients presenting with fever, jaundice and acute renal failure, there should be a high index of suspicion for malaria even in the face of negative blood film. Early and prompt diagnosis along with anti-malarial therapy, are the main measures likely to reduce the malarial ARF in this setting. Early referral of malarial ARF patients for dialysis in complicated malarial cases may further reduce mortality due to end organ failure and enhance recovery. Renal impairment typically manifests with oliguria, associated with altered renal function test are probable outcomes of plasmodium falciparum infection. plasmodium Falciparum \& other species impact on renal dysfunction needs large scale study for definite conclusion.

\section{Acknowledgement}

We, all very much thankful to the patients, though they are known or unknown to us; we dedicate this valuable work to them. We deeply thankful to Medical Director, Dean, Medical Superintendent, HOD,s and Faculties of all departments who referred patients for laboratory investigations. Very much thanks to technical staff, nurses \& phlebotomist of the hospital and specially $\mathrm{Dr}$ Sakharam Muley, Bio-statistician, Mumbai University for data analysis 


\section{References}

[1] Mishra S.K., Mohapatra S., Mohanty S., Patel N.C.,Mohapatra D.N. (2002) Journal Indian Academy of Clinical Medicine, 3 (2):141-147.

[2] Boonpucknaviq V., Sitprija V. (1979) Kidney International, 6:44-54

[3] Sitprija V. (1988) Kidney International, 34:867-877.

[4] Barsoum R.S. (2000) Journal of American Society of Nephrology, 11:2147-2154.

[5] Naqvi R., Ahmad E., Akhtar F., Naqvi A,. Rizvi A. (2003) Nephrology Dialysis Transplant, 18:1820-1823.

[6] Wilairatana P., Looareesuwan S., Charoenlarp P. (1994) Tropical Medicine and Parasitology, 45:298-300.

[7] Ogbadoyi E.O,. Tembeng F.C. (1999) Journal of Protozoology Research, 9:49-52.

[8] Weber M.W., Zimmer-Mann U. Van Hens Brock M.B., Frenkil J., Palmer A., Ehrich J.H.H., GreenWood B.M. (1999) Tropical Medicine and International Health, 4:350-394.

[9] Mishra S.K., Mohapatra S., Mohanty S. (2003) Journal Indian Academy of Clinical Medicine, 4:12-13.

[10]Sharma S.K., Sharma B.H.K., Shakya K., Khanal B., Khaniya S., Shrestha N., Parajuli K., Rijal S., Karki P. (2004) Journal of Nepal Medical Association, 43:7-9.

[11]Ogbadoyi EO, Gabi B. (2007) African Journal of Infectious Diseases, 1(1):57-64.

[12]WHO. (2000) Severe falciparum malaria. Transactions of the Royal Society of Tropical Medicine and Hygiene. 2000;94 (suppl 1):1-90.

[13]Kumar S., Melzer M., Dodds P., Watson J., Ord R. Scand (2007) J Infect Dis , 39: 255-6.

[14]Ozen M., Gungor S., Atambay M., Daldal N. (2006) Ann TropPaediatr, 26: 141-4.

[15]Kochar D.K., Saxena V., Singh N., Kochar S.K., Vijay Kumar S., Das A. (2005) www.cdc.gov/eid. V 11, No. 1.

[16]Philips R.E., White N.J., Looareesuwan S. et al. (1984) XI InternationalCongress for Tropical Medicine and Malaria, Calgary, Canada,.

[17]Saroj K. Mishra, Shradhanand Mohapatra, Sanjib Mohanty, Patel NC, Mohapatra DN. (2002) JIACM, 3(2): 141-7

[18]Barsoum R., Sitprija V. (1996) Diseases of the kidney, 222168.

[19]Eiam-Ong S, Sitprija V. (1998) Am J Kidney Dis, 32:361-75.

[20]Barsoum RS. (2000) J Am Soc Nephrol, 11: 2147-54.

[21]Gilles H.M., Hendrichse R.G. (1963) Br Med J, 2: 27.

[22]Pakasa M., Van Damme B., Desmet V.J. (1985) Br J Exp Pathol , 66: 493.

[23]Das BS. (2008) Renal failure in malaria. J Vector Borne Dis, pp. 83-97.

[24]Pakasa M., Van Damme B., Desmet V.J. (1985) Br J Exp Pathol, 66: 493.

[25]Barsoum R. (1998) Nephrol Dial Transplant 1, 13:1488-597.

[26]Howell Devid (2002) Duxbury. Pp 324-325 ISBN 0534-37770$X$.

[27]Lomax, Richard G. (2007) Statistical Concepts: A Second Course, p. 10, 0-8058-5850-4.

[28]"Null hypothesis definition". Businessdictionary.com. Retrieved 2010-07-29.

[29]Thadhani R., Pascual M., Bonventre J.V. (1996) N Engl J Med,
334:1448-60.

[30]Liano F., Pascual J. (1998) Semin Nephrol., 18:541-540

[31]Levey A.S. (1990) Kidney Int. , 38:167-184.

[32]Brivet F.G., Kleinknecht D.J., Loirat P., Landais P.J. (1996) Critical Care Med., 24:192-198.

[33]Paganani E.P., Halstenberg W.K., Goormastic M. (1996) Clin Nephrol., 46:206-211. 